\title{
Pediatric Conditions Requiring Minimal Intervention or Observation After Interfacility Transfer
}

Corrie E McDaniel, DO',2*, JoAnna Leyenaar, MD, PhD, MPH ${ }^{3,4}$, Erin Sullivan, MPH², Sanyukta Desai, MD, MSc 1,2, Larry Kessler, ScD 2,5

${ }^{1}$ Department of Pediatrics, University of Washington, Seattle, Washington; ${ }^{2}$ Seattle Children's Research Institute, Seattle, Washington; ${ }^{3}$ Department of Pediatrics, Dartmouth-Hitchcock Medical Center, Lebanon, New Hampshire; ${ }^{4}$ The Dartmouth Institute for Health Policy and Clinical Practice, Geisel School of Medicine at Dartmouth, Hanover, New Hampshire; ${ }^{5}$ Department of Health Services, University of Washington, Seattle, Washington.

Increasing regionalization of pediatric care has led to interfacility transfer of children with general pediatric conditions at rates similar to those of high-risk adults, which may delay appropriate treatment. We sought to identify common medical diagnoses that did not require significant advanced intervention and that had high rates of discharge within 1 day of interfacility transfer. Using the Pediatric Health Information System (PHIS) database, we identified all transfers into PHIS-participating children's hospitals in 2019. We excluded encounters for mental health, labor/maternity, primary newborn diagnoses, and direct admissions to an intensive care unit. Eligible encounters were categorized by duration of hospitalization and basic vs advanced intervention after transfer. Of 286,905 transfers, $197,386(68.6 \%)$ met inclusion criteria. Cough, febrile seizures, croup, and allergic reactions required advanced interventions $<10 \%$ of the time, and patients with these diagnoses were most commonly discharged within 1 day after transfer. These conditions are potential targets for building pediatric capacity in non-pediatric hospitals. Journal of Hospital Medicine 2021;16:412-415. (C) 2021 Society of Hospital Medicine
$\mathrm{R}$ egionalization of pediatric acute care is increasing across the United States, with rates of interfacility transfer for general medical conditions in children similar to those of high-risk conditions in adults. ${ }^{1}$ The inability for children to receive definitive care (ie, care provided to conclusively manage a patient's condition without requiring an interfacility transfer) within their local community has implications on public health as well as family function and financial burden. ${ }^{1,2}$ Previous studies demonstrated that $30 \%$ to $80 \%$ of interfacility transfers are potentially unnecessary, ${ }^{3-6}$ as indicated by a high proportion of short lengths of stay after transfer. While rapidity of discharge is an important factor in identifying potentially unnecessary transfers, many of these studies included diagnoses requiring specialized imaging or surgical interventions, which may not be available in referring institutions.

To highlight conditions that referring hospitals may prioritize for pediatric capacity building, we aimed to identify the most common medical diagnoses among pediatric transfer patients that did not require advanced evaluation or intervention and that had high rates of discharge within 1 day of interfacility transfer.

\section{METHODS}

We conducted a retrospective, cross-sectional, descriptive study using the Pediatric Health Information System (PHIS) da-

*Corresponding Author: Corrie McDaniel, DO; Email: Corrie.mcdaniel@ seattlechildrens.org; Telephone: 206-818-9821; Twitter: @Corrie.Erin.

Published online first June 16, 2021

Find additional supporting information in the online version of this article.

Received: February 25, 2021; Revised: May 11, 2021; Accepted: May 18, 2021

() 2021 Society of Hospital Medicine DOI 10.12788/jhm.3656 tabase, which contains administrative data from 48 geographically diverse US children's hospitals.

We included children $<18$ years old who were transferred to a participating PHIS hospital in 2019, including emergency department (ED), observation, and inpatient encounters. We identified patients through the source-of-admission code labeled as "transfer." Diagnoses were identified through the International Classification of Diseases, Tenth Revision (ICD-10) codes using the Pediatric Clinical Classification System. ${ }^{7}$ We excluded the following categories: mental or behavioral health diagnoses, maternal or labor diagnoses, primary newborn birth diagnoses, and transfers directly to an intensive care unit (ICU).

For each diagnosis, we determined the number of transfers and frequency of rapid discharge, defined as either discharge from the ED without admission or admission and discharge within 1 day from a general inpatient unit. As discharge times are not reliably available in PHIS, all patients discharged on the day of transfer or the following calendar day were identified as rapid discharge. Medical complexity was determined through applying the Pediatric Medical Complexity Algorithm (PMCA). ${ }^{8}$

To identify diagnoses seen with sufficient frequency to represent potentially useful areas for referring hospitals to target, we limited our analysis to diagnoses that had a minimum of 576 transfers per year, equivalent to at least 1 transfer for that diagnosis per month per PHIS hospital. We then categorized the frequency of interventions after transfer, including (1) no interventions received; (2) basic interventions only, defined as receiving any intravenous fluids, antimicrobials, antipyretics or analgesics, and/or basic imaging (ie, radiography and computed tomography [CT]); or (3) advanced interventions, including transfer to an ICU after initial presentation/management in the ED or inpatient 
TABLE. Medical Diagnoses Most Commonly Discharged Rapidly After Interfacility Transfer

\begin{tabular}{|c|c|c|c|c|c|c|}
\hline \multirow[b]{2}{*}{$\begin{array}{l}\text { Diagnosis group } \\
\text { (total No. transferred in 2019a) }\end{array}$} & \multicolumn{3}{|c|}{$\begin{array}{l}\text { Discharged rapidly, } \\
\text { No. }(\%)\end{array}$} & \multicolumn{3}{|c|}{$\begin{array}{l}\text { Interventions received, } \\
\text { No. (\%) }\end{array}$} \\
\hline & Total & $\begin{array}{l}\text { Emergency } \\
\text { department }\end{array}$ & $\begin{array}{l}\text { Within } 1 \text { calendar } \\
\text { day of admission }\end{array}$ & $\begin{array}{l}\text { None } \\
\text { received }\end{array}$ & $\begin{array}{c}\text { Any basic } \\
\text { intervention }\end{array}$ & $\begin{array}{l}\text { Any advanced } \\
\text { intervention }^{d}\end{array}$ \\
\hline Cough (620) & $611(98.5)$ & $582(93.9)$ & $29(4.7)$ & $412(66.5)$ & $207(33.4)$ & $8(1.3)$ \\
\hline Head injury, unspecified (655) & $642(98.0)$ & $596(91.0)$ & $46(7.0)$ & $448(68.4)$ & $194(29.6)$ & $58(8.9)$ \\
\hline Nonspecific chest pain (603) & $579(96.0)$ & $473(78.4)$ & $106(17.6)$ & $272(45.1)$ & $291(48.3)$ & $107(17.7)$ \\
\hline Superficial injury; contusion $(1,712)$ & $1,629(95.2)$ & $1,437(83.9)$ & $192(11.2)$ & $1,070(62.5)$ & $616(36.0)$ & $147(8.6)$ \\
\hline Abdominal pain $(3,392)$ & $3,121(92.0)$ & $2,523(74.4)$ & $598(17.6)$ & $753(22.2)$ & $1,973(58.2)$ & $1,912(56.4)$ \\
\hline Open wounds of head; neck; and trunk $(2,424)$ & $2,229(92.0)$ & $1,887(77.8)$ & $342(14.1)$ & $1,515(62.5)$ & $885(36.5)$ & $488(20.1)$ \\
\hline Simple febrile seizures (584) & $536(91.8)$ & $342(58.6)$ & $194(33.2)$ & $410(70.2)$ & $167(28.6)$ & $44(7.5)$ \\
\hline Other injuries and conditions due to external causes $(1,543)$ & $1,407(91.2)$ & $1,190(77.1)$ & $217(14.1)$ & $637(41.3)$ & $876(56.8)$ & $205(13.3)$ \\
\hline Syncope (799) & $713(89.2)$ & $477(59.7)$ & $236(29.5)$ & $432(54.1)$ & $314(39.3)$ & $166(20.8)$ \\
\hline Nausea and vomiting $(1,676)$ & $1,486(88.7)$ & $1,284(76.6)$ & $202(12.1)$ & $766(45.7)$ & $772(46.1)$ & $463(27.6)$ \\
\hline Croup $(2,169)$ & 1,893 (87.3) & $882(40.7)$ & $1,011(46.6)$ & $1,559(71.9)$ & $581(26.8)$ & $143(6.6)$ \\
\hline Allergic reactions $(1,548)$ & $1,350(87.2)$ & $949(61.3)$ & $401(25.9)$ & $1,192(77.0)$ & $334(21.6)$ & $94(6.1)$ \\
\hline Other nontraumatic joint disorders (754) & $647(85.8)$ & $546(72.4)$ & $101(13.4)$ & $104(13.8)$ & $629(83.4)$ & $205(27.2)$ \\
\hline Gastroenteritis and colitis, noninfectious $(1,296)$ & $1,104(85.2)$ & $856(66.0)$ & $248(19.1)$ & $658(50.8)$ & $587(45.3)$ & $253(19.5)$ \\
\hline Acute upper respiratory infection $(3,510)$ & $2,964(84.4)$ & $2,349(66.9)$ & $615(17.5)$ & $2,486(70.8)$ & $1,005(28.6)$ & $207(5.9)$ \\
\hline Open wounds of extremities (977) & $824(84.3)$ & $676(69.2)$ & $148(15.1)$ & $345(35.3)$ & $630(64.5)$ & $242(24.8)$ \\
\hline Fever of unknown origin $(2,784)$ & $2,344(84.2)$ & $1,974(70.9)$ & $370(13.3)$ & $1,530(55.0)$ & $1,196(43.0)$ & $300(10.8)$ \\
\hline Foreign body in alimentary tract $(1,426)$ & $1,182(82.9)$ & $499(35.0)$ & $683(47.9)$ & $133(9.3)$ & $1,261(88.4)$ & $796(55.8)$ \\
\hline Poisoning by other medications and drugs (849) & $701(82.6)$ & $167(19.7)$ & $534(62.9)$ & $451(53.1)$ & $380(44.8)$ & $108(12.7)$ \\
\hline Viral infection $(4,263)$ & $3,391(79.5)$ & $2,773(65.0)$ & $618(14.5)$ & $2,654(62.3)$ & $1,540(36.1)$ & $436(10.2)$ \\
\hline \multicolumn{7}{|c|}{ a Limited to diagnoses with at least 576 transfers from January through December 2019.} \\
\hline \multicolumn{7}{|c|}{ b Rapid discharge refers to patients discharged from the emergency department or admitted patients who were discharged within 1 calendar day. } \\
\hline \multicolumn{7}{|c|}{ "Basic intervention was defined as the receipt of (1) radiography, and/or computed tomography (CT), (2) intravenous (IV) fluids, (3) IV analgesics or antipyretics, and/or (4) IV antibiotics. } \\
\hline \multicolumn{7}{|c|}{$\begin{array}{l}\text { ¿Advanced intervention was defined as the receipt of (1) imaging including magnetic resonance (MR) imaging, MR angiography or venography, CT angiography, and/or ultrasound; (2) admis- } \\
\text { sion to an intensive care unit after hospitalization; and/or (3) an operative procedure. }\end{array}$} \\
\hline
\end{tabular}

ward, advanced imaging (eg, ultrasound, magnetic resonance [MR] imaging, MR angiography or venography, CT angiography), or any surgical intervention. A full categorization of basic and advanced interventions is available in Appendix Table 1.

For descriptive statistics, we calculated means for normally distributed variables, medians for continuous variables with nonnormal distributions, and percentages for binary variables. Comparisons were made using t-tests and chi-square tests.

This study was approved by the Seattle Children's Institutional Review Board.

\section{RESULTS}

We identified 286,905 transfers into participating PHIS hospitals in 2019. Of these, 89,519 (31.2\%) were excluded (Appendix Table 2), leaving 197,386 (68.6\%) transfers. Patients discharged within 1 day were more likely to have public or unknown insurance $(65.1 \%$ vs $61.5 \%, P<0.01)$, to have no co-occurring chronic conditions $(60.2 \%$ vs $28.5 \%, P<0.01)$, and to reside within the Northeast (35.0\% vs 11.0\%, $P<0.01$ ) (Appendix Table 3).

The most common medical diagnoses among these transfers included acute bronchiolitis (4.3\% of all interfacility transfers, $n=8,425)$, chemotherapy $(4.0 \%, n=7,819)$, and asthma (3.3\%, $n=6,430$ ) (Appendix Table 4); $45.9 \%$ of bronchiolitis, $15.0 \%$ of chemotherapy, and $67.4 \%$ of asthma transfers were rapidly discharged.

The Table shows the medical conditions among transfers that most frequently experienced rapid discharge (primary surgical diagnoses are presented in Appendix Table 5). Within this cohort, patients transferred for cough were most likely to be rapidly discharged, with $98.5 \%(n=611)$ discharged within 
1 day of transfer. Among these, $66.5 \%(n=412)$ received no interventions and $33.4 \%(n=207)$ received only basic interventions. Only $1.3 \%(n=8)$ required any advanced intervention. Similarly, $96.0 \%(n=603)$ of patients with the diagnosis of chest pain were rapidly discharged, with $45.1 \%(n=272)$ requiring no interventions, $48.3 \%$ ( $n=291$ ) receiving basic interventions, and $17.7 \%(n=107)$ requiring advanced intervention. Patients with the diagnosis of febrile seizures, croup, and allergic reactions were rapidly discharged $91.8 \%(n=584), 87.3 \%(n=1,893)$ and $87.2 \%$ ( $n=1,350)$ of the time, respectively, and more than $70 \%$ patients with these diagnoses underwent no intervention after transfer. In addition, while $92.0 \%$ ( $n=3,392$ ) of patients with abdominal pain diagnoses were discharged rapidly, $55.5 \%$ ( $n=1,883$ ) received advanced imaging (Appendix Table 6). Similarly, while $92.0 \%$ ( $n=2,229)$ of patients with open wounds to the head, neck, and trunk were discharged rapidly, $17.3 \%$ $(n=419)$ of patients with these diagnoses required a surgical intervention after transfer (Appendix Table 6).

\section{DISCUSSION}

We have identified medical conditions that not only had high rates of rapid discharge after transfer, but also received minimal intervention from the accepting institution. Although bronchiolitis and chemotherapy were the most common conditions for which patients were transferred, the range of severity varied widely, with more than $50 \%$ of bronchiolitis and $85 \%$ of chemotherapy transfers requiring hospitalization for longer than 1 day. Diagnoses such as chemotherapy, although common among transferred patients, likely represent conditions that may not be appropriate to care for in pediatric-limited settings, as they require subspecialized pediatric care. General conditions, however, such as cough, chest pain, allergic reactions, and febrile seizures may represent diagnoses for which it would be appropriate for general hospitals to develop infrastructure to provide definitive pediatric care given how infrequently specialized pediatric resources are needed in caring for these conditions.

Identifying conditions as potential targets to reduce the number of interfacility transfers requires balancing a hospital's capacity (or lack thereof) for pediatric admissions, perceived risk of decompensation, referring provider discomfort, and parental preference. ${ }^{9-11}$ Although several studies have identified conditions associated with frequent transfer and rapid discharge, ${ }^{3-5}$ prior studies' conclusions that $40 \%$ or more of interhospital transfers may be avoidable are potential overestimates, representing conditions that may not be appropriate to care for in pediatric-limited settings given their need for advanced interventions. Our findings demonstrate that defining a cohort of conditions based on frequency of transfer, even when accounting for minimal intervention post transfer, may not adequately capture avoidable transfers. For example, abdominal pain was one of the conditions for which patients were most frequently transferred, with $92 \%$ of patients discharged rapidly. However, the most common surgical transfer was acute appendicitis with peritonitis. Many of these transfers may have been identified initially as "abdominal pain" at the referring institution, highlighting the role of diagnostic uncertainty in identifying preventable transfers. In addition, more than $56 \%$ of patients transferred for abdominal pain required advanced interventions, further illustrating the potential risk and uncertainty for referring hospitals that do not have the capacity for advanced imaging or surgical intervention.

The rapid upscale of telehealth may provide a unique opportunity to support the provision of pediatric care within local communities. ${ }^{12,13}$ As many general hospitals do not have ultrasound technicians trained for children available 24 hours per day, several conditions that fell into the advanced intervention category, like abdominal pain, were driven by the receipt of an ultrasound at the accepting hospital. Targeted work to expand ultrasound capabilities at referring hospitals may enable changing the categorization of an ultrasound to a basic intervention rather than an advanced intervention. Paired with telehealth, this might broaden the scope of potential diagnoses that could be triaged to stay within referring institutions.

Building infrastructure to prevent interfacility transfers may improve healthcare access for children in rural areas proportionately more than children in urban areas. Children in rural communities experience significantly higher rates of interfacility transfers than children in urban areas. ${ }^{14}$ This increases financial burden and causes additional distress and inconvenience for families. ${ }^{15}$ With constraints in staffing capacity, equipment, and finances, identifying a subset of medical conditions is a critical initial step to inform the design of targeted interventions to support pediatric healthcare delivery in local communities and avoid costly transfers, although it is not the wholesale solution. Additional utilization of tools such as informed shared decision-making resources and implementation of pediatric-specific protocols likely represent additional necessary steps.

Our study has several limitations. Because we used administrative data, there is a risk of misclassifying diagnoses. We attempted to mitigate this by using a standard ICD-10-based, pediatric-specific grouper. ICD-10 coding is also based upon discharge diagnoses, which inherently has retrospective bias that cannot capture the diagnostic uncertainty when making an initial decision for transfer. In addition, without a comparator group of patients who were not transferred, it remains unknown to what extent balancing factors informed the decision to transfer or whether these diagnoses represent conditions that the referring hospital encounters only a few times a year, or alternatively, that the percentage transferred represents a small fraction of the referring institution's population with a given diagnosis.

\section{CONCLUSION}

Our exploration of pediatric interfacility transfers that experienced rapid discharge with minimal intervention provides a building block to support the provision of definitive pediatric care in non-pediatric hospitals and represents a step towards addressing limited access to care in general hospitals.

Disclosures: The authors have no conflicts of interest to disclose. 


\section{References}

1. França UL, McManus ML. Availability of definitive hospital care for children JAMA Pediatr. 2017;171(9):e171096. https://doi.org/10.1001/jamapediatrics.2017.1096

2. Mumford V, Baysari MT, Kalinin D, et al. Measuring the financial and productivity burden of paediatric hospitalisation on the wider family network. $J$ Paediatr Child Health. 2018;54(9):987-996. https://doi.org/10.1111/jpc.13923

3. Richard KR, Glisson KL, Shah N, et al. Predictors of potentially unnecessary transfers to pediatric emergency departments. Hosp Pediatr. 2020;10(5):424429. https://doi.org/10.1542/hpeds.2019-0307

4. Gattu RK, Teshome G, Cai L, Wright C, Lichenstein R. Interhospital pediatric patient transfers-factors influencing rapid disposition after transfer. Pediatr Emerg Care. 2014;30(1):26-30. https://doi.org/10.1097/PEC.0000000000000061

5. Li J, Monuteaux MC, Bachur RG. Interfacility transfers of noncritically ill children to academic pediatric emergency departments. Pediatrics. 2012;130(1):83-92. https://doi.org/10.1542/peds.2011-1819

6. Rosenthal JL, Lieng MK, Marcin JP, Romano PS. Profiling pediatric potentially avoidable transfers using procedure and diagnosis codes. Pediatr Emerg Care. 2019 Mar 19;10.1097/PEC.0000000000001777. https://doi.org/10.1097/ PEC.0000000000001777

7. Pediatric clinical classification system (PECCS) codes. Children's Hospital Association. December 11, 2020. Accessed June 3, 2021. https://www.childrenshospitals.org/Research-and-Data/Pediatric-Data-and-Trends/2020/ Pediatric-Clinical-Classification-System-PECCS

8. Simon TD, Haaland W, Hawley K, Lambka K, Mangione-Smith R. Development and validation of the pediatric medical complexity algorithm (PMCA) version 3.0. Acad Pediatr. 2018;18(5):577-580. https://doi.org/10.1016/j. acap.2018.02.010
9. Rosenthal JL, Okumura MJ, Hernandez L, Li ST, Rehm RS. Interfacility transfers to general pediatric floors: a qualitative study exploring the role of communication. Acad Pediatr. 2016;16(7):692-699. https://doi.org/10.1016/j. acap.2016.04.003

10. Rosenthal JL, Li ST, Hernandez L, Alvarez M, Rehm RS, Okumura MJ. Familial caregiver and physician perceptions of the family-physician interactions during interfacility transfers. Hosp Pediatr. 2017;7(6):344-351. https://doi. org/10.1542/hpeds.2017-0017

11. Peebles ER, Miller MR, Lynch TP, Tijssen JA. Factors associated with discharge home after transfer to a pediatric emergency department. Pediatr Emerg Care. 2018;34(9):650-655. https://doi.org/10.1097/ PEC.0000000000001098

12. Labarbera JM, Ellenby MS, Bouressa P, Burrell J, Flori HR, Marcin JP. The impact of telemedicine intensivist support and a pediatric hospitalist program on a community hospital. Telemed J E Health. 2013;19(10):760-766. https:// doi.org/10.1089/tmj.2012.0303

13. Haynes SC, Dharmar M, Hill BC, et al. The impact of telemedicine on transfer rates of newborns at rural community hospitals. Acad Pediatr. 2020;20(5):636641. https://doi.org/10.1016/j.acap.2020.02.013

14. Michelson KA, Hudgins JD, Lyons TW, Monuteaux MC, Bachur RG, Finkelstein JA. Trends in capability of hospitals to provide definitive acute care for children: 2008 to 2016. Pediatrics. 2020;145(1). https://doi.org/10.1542/ peds.2019-2203

15. Mohr NM, Harland KK, Shane DM, Miller SL, Torner JC. Potentially avoidable pediatric interfacility transfer is a costly burden for rural families: a cohort study. Acad Emerg Med. 2016;23(8):885-894. https://doi. org/10.1111/acem.12972 